SOGANG-HEP 298/02

hep-th/0206001

\title{
Propagators in Noncommutative Instantons
}

\author{
Bum-Hoon Lee ${ }^{a}$ and Hyun Seok Yang b̆ \\ ${ }^{a}$ Department of Physics, Sogang University, Seoul 121-742, Korea \\ ${ }^{b}$ Department of Physics, National Taiwan University, Taipei 106, Taiwan, R.O.C.
}

\begin{abstract}
We explicitly construct Green functions for a field in an arbitrary representation of gauge group propagating in noncommutative instanton backgrounds based on the ADHM construction. The propagators for spinor and vector fields can be constructed in terms of those for the scalar field in noncommutative instanton background. We show that the propagators in the adjoint representation are deformed by noncommutativity while those in the fundamental representation have exactly the same form as the commutative case.
\end{abstract}

PACS numbers: 11.15.-q, 11.15.Tk, 02.40.Gh

September 12, 2018

*bhl@ccs.sogang.ac.kr

†hsyang@phys.ntu.edu.tw 


\section{Introduction}

Instantons were found by Belavin, Polyakov, Schwartz and Tyupkin (BPST) [1] almost thirty years ago, as topologically nontrivial solutions of the duality equations of the Euclidean YangMills theory with finite action. Immediately instantons were realized to describe the tunnelling processes between different $\theta$-vacua in Minkowski space and lead to the strong CP problem in QCD [2, 阿. (For the earlier development of instanton physics, see the collection of papers [4.) The non-perturbative chiral anomaly in the instanton background led to baryon number violation and a solution to the $U(1)$ problem [5, 6]. These revealed that instantons can have their relevance to phenomenological models like QCD and the Standard model [7].

Instanton solutions also appear as BPS states in string theory. They are described by Dp-branes bound to $\mathrm{D}(\mathrm{p}+4)$-branes [8, 9]. Subsequently, in [10, 11], low-energy excitations of D-brane bound states were used to explain the microscopic degrees of freedom of blackhole entropy, for which the information on the instanton moduli space has a crucial role. In addition the multi-instanton calculus was used for a non-perturbative test of AdS/CFT correspondence [12, 13, 14, 15], where the relation between Yang-Mills instantons and D-instantons was beautifully confirmed by the explicit form of the classical D-instanton solution in $\operatorname{Ad} S_{5} \times \mathbf{S}^{5}$ background and its associated supermultiplet of zero modes.

Recently instanton solutions on noncommutative spaces have been turned out to have more richer spectrums. While commutative instantons are always BPS states, noncommutative instantons admit both BPS and non-BPS states. Especially, instanton solutions can be found in $U(1)$ gauge theory and the moduli space of non-BPS instantons is smooth, small instanton singularities being resolved by the noncommutativity [16, [17]. Remarkably, instanton solutions in noncommutative gauge theory can also be studied by Atiyah-Drinfeld-Hitchin-Manin (ADHM) equation [18] slightly modified by the noncommutativity [16]. ADHM construction uses some quadratic matrix equations, hence noncommutative objects in nature, to construct (anti-)selfdual configurations of the gauge field. Thus the noncommutativity of space doesn't make any serious obstacle for the ADHM construction of noncommutative instantons and indeed it turns out that it is a really powerful tool even for noncommutative instantons. Recently much progress has been made in this direction [16, 17, 19, 20, 21, 22, 23, 24, 25, 26, 27, 28, 29, 30, 31, 32, 33, 34, 35].

In order to calculate instanton effects in quantum gauge theory, it is important to know the Green function in instanton backgrounds [6]. In this paper, based on the ADHM construction, we will construct the Green functions for a field in an arbitrary representation of gauge group propagating in noncommutative instanton backgrounds. Recently several papers [26, 36, 37, 38, 39, 40, 41, 42, 43] discussed the instanton moduli space and the instanton calculus in noncommutative spaces. This paper is organized as follows. In next section we review briefly the Weyl ordering prescription for operators and the Green function in noncommutative space, needed for later applications. In Section 3, we generalize the argument in [44] 
to noncommutative space and show that the propagators for spinor and vector fields can be constructed in terms of those for the scalar field in noncommutative instanton background. In Section 4, we explicitly construct the scalar propagators in the fundamental representation of $G$ and the tensor product $G_{1} \times G_{2}$ [45] where the adjoint representation is a special case. We observe that the propagator in the adjoint representation or the tensor product gauge group $G_{1} \times G_{2}$ is deformed by noncommutativity while that in the fundamental representation has exactly the same form as the commutative case. In Section 5 we speculatively discuss some important issues such as an infrared divergence in the vector propagator, the zero modes for the tensor product gauge group and conformal property of instanton propagators.

\section{Green Function in Noncommutative Space}

In this section we review briefly the Weyl ordering prescription for operators and the Green function in noncommutative space [46, 47], needed for later applications.

Here we will work in general in flat noncommutative Euclidean space $\mathbf{R}^{4}$ represented by

$$
\left[\hat{x}^{\mu}, \hat{x}^{\nu}\right]=i \theta^{\mu \nu}
$$

where $\theta^{\mu \nu}=-\theta^{\nu \mu}$ and we use ${ }^{\wedge}$ to indicate operators in $\mathcal{A}_{\theta}$ for a moment. Since $\theta^{\mu \nu}$ is an anti-symmetric tensor, let's decompose them into self-dual and anti-self-dual parts:

$$
\theta_{\mu \nu}=\eta_{\mu \nu}^{a} \zeta^{a}+\bar{\eta}_{\mu \nu}^{a} \chi^{a}
$$

Since the self-duality condition is invariant under $S O(4)$ rotations (or more generally $S L(4, \mathbf{R}$ ) transformations), one can always make the matrix $\theta_{\mu \nu}$ to a standard symplectic form by performing the $S O(4)$ transformation $R$ :

$$
\theta=R \tilde{\theta} R^{T}
$$

where we choose $\tilde{\theta}$ as

$$
\tilde{\theta}_{\mu \nu}=\left(\begin{array}{cccc}
0 & \theta_{1} & 0 & 0 \\
-\theta_{1} & 0 & 0 & 0 \\
0 & 0 & 0 & \theta_{2} \\
0 & 0 & -\theta_{2} & 0
\end{array}\right) .
$$

There are four important cases to consider:

$$
\begin{aligned}
& -\theta_{1}=\theta_{2}=0: \quad \text { commutative } \mathbf{R}^{4}, \\
& \text { - } \theta_{1}=\theta_{2}=\frac{\zeta}{4}: \quad \text { self-dual } \mathbf{R}_{N C}^{4}, \\
& \text { - } \theta_{1}=-\theta_{2}=\frac{\zeta}{4}: \quad \text { anti-self-dual } \mathbf{R}_{N C}^{4}, \\
& \text { - } \theta_{1} \theta_{2}=0 \text { but } \theta_{1}+\theta_{2}=\frac{\zeta}{2}: \quad \mathbf{R}_{N C}^{2} \times \mathbf{R}_{C}^{2} .
\end{aligned}
$$


By noncommutative space $\mathbf{R}_{N C}^{4}$ one means the algebra $\mathcal{A}_{\theta}$ generated by the $\hat{x}^{\mu}$ satisfying (2.1). The commutation relation (2.1) in the basis (2.4) is equivalent to that of the annihilation and creation operators for one dimensional or two-dimensional harmonic oscillator:

$$
\left[a_{a}, a_{b}^{\dagger}\right]=\delta_{a b},
$$

where $a=1,2$ for (2.6) and (2.7) and $a=1$ for (2.8). Explicitly, for self-dual and anti-self-dual $\mathbf{R}_{N C}^{4}$ in (2.6) and (2.7),

$$
a_{a}^{\dagger}=\sqrt{\frac{2}{\zeta}}\left(\hat{x}^{2 a}+i \epsilon^{a-1} \hat{x}^{2 a-1}\right), \quad a_{a}=\sqrt{\frac{2}{\zeta}}\left(\hat{x}^{2 a}-i \epsilon^{a-1} \hat{x}^{2 a-1}\right),
$$

where $a=1,2$ and $\epsilon=\theta_{1} / \theta_{2}$. So, for self-dual and anti-self-dual $\mathbf{R}_{N C}^{4}$, the representation space $\mathcal{H}$ of $\mathcal{A}_{\theta}$ can be identified with the Fock space $\mathcal{F}=\sum_{\left(n_{1}, n_{2}\right) \in \mathbf{Z}_{\geq 0}^{2}} \mathbf{C} \mid n_{1}, n_{2}>$, where $n_{1}, n_{2}$ are occupation numbers in the harmonic oscillators. Thus the noncommutative space $\mathbf{R}_{N C}^{4}$ in the basis $\mathcal{F}$ becomes two-dimensional integer lattice $\left\{\left(n_{1}, n_{2}\right) \in \mathbf{Z}_{\geq 0}^{2}\right\}$ and the integration on $\mathbf{R}_{N C}^{4}$ can be defined by the sum over the lattice,

$$
\operatorname{Tr}_{\mathcal{H}} \mathcal{O}(x) \equiv\left(\frac{\zeta \pi}{2}\right)^{2} \sum_{\left(n_{1}, n_{2}\right)}\left\langle n_{1}, n_{2}|\mathcal{O}(x)| n_{1}, n_{2}\right\rangle
$$

for an operator $\mathcal{O}(x)$ in $\mathcal{A}_{\theta}$. While, for $\mathbf{R}_{N C}^{2} \times \mathbf{R}_{C}^{2}$ in (2.8),

$$
a^{\dagger}=\frac{\hat{x}^{2 a}+i \hat{x}^{2 a-1}}{\sqrt{\zeta}}, \quad a=\frac{\hat{x}^{2 a}-i \hat{x}^{2 a-1}}{\sqrt{\zeta}},
$$

where $a=1$ for $\theta_{1} \neq 0$ and $a=2$ for $\theta_{2} \neq 0$. In this case, the representation space $\mathcal{H}$ is given by $\mathcal{F}=\sum_{n \in \mathbf{Z}_{\geq 0}} \mathbf{C}|n\rangle$ and the integration for an operator $\mathcal{O}(x)$ in $\mathcal{A}_{\theta}$ with $\theta_{1} \neq 0$, for example, can be replaced by

$$
\int d^{4} x \mathcal{O}(x) \rightarrow \zeta \pi \sum_{n \in \mathbf{Z} \geq 0} \int d^{2} x\langle n|\mathcal{O}(x)| n\rangle
$$

where $d^{2} x=d x^{3} d x^{4}$.

We introduce coherent states defined by

$$
|\xi\rangle=e^{\xi a^{\dagger}}|0\rangle, \quad\langle\xi|=\langle 0| e^{\bar{\xi} a}
$$

where $|0\rangle$ is a vacuum defined by $a|0\rangle=0$. For notational simplicity, we only present the construction for the algebra (2.12), but the similar construction can be given for (2.10), for which $|\xi\rangle=e^{\xi^{a} a_{a}^{\dagger}}|0\rangle$. The state $|\xi\rangle$ satisfies

$$
a|\xi\rangle=\xi|\xi\rangle, \quad\langle\xi| a^{\dagger}=\langle\xi| \bar{\xi}
$$

and

$$
\langle\eta \mid \xi\rangle=e^{\bar{\eta} \xi}, \quad \int \frac{d \bar{\xi} d \xi}{2 \pi i} e^{-|\xi|^{2}}|\xi\rangle\langle\xi|=1 .
$$


Then we see that

$$
\left\langle\eta\left|e^{i\left(k_{1} \hat{x}^{1}+k_{2} \hat{x}^{2}\right)}\right| \xi\right\rangle=e^{-\frac{\zeta k^{2}}{8}} e^{i\left(k_{1} z^{1}+k_{2} z^{2}\right)} e^{\bar{\eta} \xi}
$$

where $k^{2}=k_{1}^{2}+k_{2}^{2}$ and $z^{1}=i \frac{\sqrt{\zeta}}{2}(\xi-\bar{\eta}), z^{2}=\frac{\sqrt{\zeta}}{2}(\xi+\bar{\eta})$.

It is well-known that the Weyl or symmetric ordering prescription provides the procedure that maps commutative smooth functions onto operators acting on the Fock space $\mathcal{F}$ 48]:

$$
f(x) \longmapsto \hat{f}(\hat{x})=\int \frac{d^{4} k}{(2 \pi)^{4}} f(k) e^{i k \cdot \hat{x}},
$$

where

$$
f(k)=\int d^{4} x f(x) e^{-i k \cdot x} .
$$

Using the prescription (2.18), it is easy to show that the operator multiplication in $\mathcal{A}_{\theta}$ is isomorphic to the Moyal product of functions:

$$
\text { If } f(x) \longmapsto \hat{f}(\hat{x}) \text { and } g(x) \longmapsto \hat{g}(\hat{x}) \text {, then }(f * g)(x) \longmapsto(\hat{f} \hat{g})(\hat{x}) \text {, }
$$

where the Moyal product is defined as

$$
(f * g)(x)=\left.e^{\frac{i}{2} \theta^{\mu \nu} \frac{\partial}{\partial x^{\mu}} \frac{\partial}{\partial y^{\nu}}} f(x) g(y)\right|_{x=y} .
$$

In order to discuss instanton propagators in the noncommutative space (2.1), we first should know the free Green function $\hat{G}^{(0)}(\hat{x}, \hat{y})$ for the ordinary Laplacian [46, 47]:

$$
-\hat{\partial}_{\mu} \hat{\partial}_{\mu} \hat{G}^{(0)}(\hat{x}, \hat{y})=\hat{\delta}(\hat{x}-\hat{y})
$$

where the derivative for an operator $\hat{f}(\hat{x})$ is defined as

$$
\hat{\partial}_{\mu} \hat{f}(\hat{x})=-i\left(\theta^{-1}\right)_{\mu \nu}\left[\hat{x}^{\nu}, \hat{f}(\hat{x})\right] .
$$

In commutative $\mathbf{R}^{4}$, it is given by

$$
G^{(0)}(x, y)=\frac{1}{4 \pi^{2}(x-y)^{2}} .
$$

Here some comments should be made. In order to define the Green function, we have introduced the tensor product $\mathcal{A}_{\theta}^{1,2}=\mathcal{A}_{\theta}^{1} \otimes \mathcal{A}_{\theta}^{2}$ of two copies of the algebra $\mathcal{A}_{\theta}$. We represent $\mathcal{A}_{\theta}^{1,2}$ as an algebra of operators on the tensor product $\mathcal{H}^{1,2}=\mathcal{H}^{1} \otimes \mathcal{H}^{2}$ of two Fock spaces. The functions $\hat{G}^{(0)}(\hat{x}, \hat{y}), \hat{\delta}(\hat{x}-\hat{y}) \in \mathcal{A}_{\theta}^{1,2}$ are operators acting on $\mathcal{H}^{1,2}$. We identify $\hat{x}^{\mu}=\hat{x}^{\mu} \otimes 1$ and $\hat{y}^{\mu}=1 \otimes \hat{y}^{\mu}$ in the tensor product. Thus in operator sense $\left[\hat{x}^{\mu}, \hat{y}^{\nu}\right]=0$. T Therefore, if we introduce the "center of mass coordinates" $\hat{R}^{\mu}$ and the "relative coordinates" $\hat{r}^{\mu}$ defined by

$$
\hat{R}^{\mu}=\frac{\hat{x}^{\mu}+\hat{y}^{\mu}}{2}, \quad \hat{r}^{\mu}=\hat{x}^{\mu}-\hat{y}^{\mu},
$$

\footnotetext{
${ }^{1}$ This is consistent with the fact that the Moyal bracket between two sets of independent variables vanishes, that is $x^{\mu} * y^{\nu}-y^{\nu} * x^{\mu}=0$ since $\frac{\partial y^{\nu}}{\partial x^{\mu}}=0$.
} 
they satisfy the following commutation relations 46]

$$
\left[\hat{R}^{\mu}, \hat{R}^{\nu}\right]=\frac{i}{2} \theta^{\mu \nu}, \quad\left[\hat{r}^{\mu}, \hat{r}^{\nu}\right]=2 i \theta^{\mu \nu}, \quad\left[\hat{R}^{\mu}, \hat{r}^{\nu}\right]=0 .
$$

The tensor product $\mathcal{A}_{\theta}^{1,2}$ can be thus decomposed in the form

$$
\mathcal{A}_{\theta}^{1,2} \cong \mathcal{D} \otimes \mathcal{R}
$$

where $\hat{R}^{\mu}$ acts on $\mathcal{D}$ and $\hat{r}^{\mu}$ on $\mathcal{R}$. Since the noncommutative space (2.1) is homogeneous and so always respects a global translation symmetry, it is reasonable to require the translation invariance for the Green function $\hat{G}^{(0)}(\hat{x}, \hat{y})$. In other words the Green function depends only on $\hat{r}^{\mu}$. Using the Weyl prescription (2.18), we see that 2

$$
\begin{aligned}
& G^{(0)}(x-y)=\int \frac{d^{4} k}{(2 \pi)^{4}} G^{(0)}(k) e^{i k \cdot(x-y)}, \\
& \delta(x-y)=\int \frac{d^{4} k}{(2 \pi)^{4}} e^{i k \cdot(x-y)} .
\end{aligned}
$$

Then the defining relation (2.22) implies that $G^{(0)}(k)=\frac{1}{k^{2}}$.

To discuss more general Green functions, especially instanton propagators, let's describe a formal procedure defining the Green function. Let $\Delta$ be a linear operator on $\mathcal{A}_{\theta}$ with a set of eigenvectors $\phi_{r}(x) \in \mathcal{A}_{\theta}$ and corresponding eigenvalues $\lambda_{r}$ :

$$
\Delta \phi_{r}(x)=\lambda_{r} \phi_{r}(x)
$$

where the parameter $r$ can be either continuous or discrete. We shall assume the completeness of $\phi_{r}(x)$

$$
\operatorname{Tr}_{\mathcal{H}} \phi_{r}(x)^{\dagger} \phi_{s}(x)=\delta_{r s}
$$

in the Hilbert space $\mathcal{H}_{p}$ of one-particle states to be

$$
\mathcal{H}_{p}=\left\{\phi(x)=\sum_{r} a_{r} \phi_{r}(x): \sum_{r}\left|a_{r}\right|^{2}<\infty\right\} .
$$

As usual the $a_{r}$ becomes operators (for example, creation or annihilation operators of a particle with quantum number $r$ ) when the field is quantized. The Green function is defined as the formal sum

$$
G(x, y)=\sum_{r} \lambda_{r}^{-1} \phi_{r}(x) \phi_{r}(y)^{\dagger} .
$$

For the free Green function in (2.22), for example, $\phi_{k}(x)=e^{i k \cdot x}$ and $\lambda_{k}=k^{2}$ for the Laplacian $\Delta=-\partial_{\mu} \partial_{\mu}$. In this case, the sum over $r$ should be the integration over momenta $k^{\mu}$ as in (2.28).

\footnotetext{
${ }^{2}$ From now on, we will delete ${ }^{\wedge}$ to indicate operators in $\mathcal{A}_{\theta}$ for notational convenience as long as it doesn't cause any confusion.
} 


\section{Instanton Propagators in Noncommutative Space}

In this section we will generalize the argument in [44 to noncommutative space to construct propagators for spinor and vector fields in terms of those for the scalar field in noncommutative instanton background. This generalization is straightforward so one may regard it as a review of Sec. II and Sec. III in 44]. This result definitely generalizes that of free fields 49; the Green functions for spinor and vector fields propagating in vacuum are determined by the corresponding scalar propagator.

To consider spinor propagator, let's introduce quaternions defined by

$$
\mathbf{x}=x_{\mu} \sigma^{\mu}, \quad \overline{\mathbf{x}}=x_{\mu} \bar{\sigma}^{\mu}
$$

where $\sigma^{\mu}=\left(i \tau^{a}, 1\right)$ and $\bar{\sigma}^{\mu}=\left(-i \tau^{a}, 1\right)=-\sigma^{2} \sigma^{\mu T} \sigma^{2}$. The quaternion matrices $\sigma^{\mu}$ and $\bar{\sigma}^{\mu}$ have the basic properties

$$
\begin{array}{ll}
\sigma^{\mu} \bar{\sigma}^{\nu}=\delta^{\mu \nu}+i \sigma^{\mu \nu}, & \sigma^{\mu \nu}=\eta_{\mu \nu}^{a} \tau^{a}=* \sigma^{\mu \nu} \\
\bar{\sigma}^{\mu} \sigma^{\nu}=\delta^{\mu \nu}+i \bar{\sigma}^{\mu \nu}, & \bar{\sigma}^{\mu \nu}=\bar{\eta}_{\mu \nu}^{a} \tau^{a}=-* \bar{\sigma}^{\mu \nu}
\end{array}
$$

and

$$
\begin{aligned}
& \bar{\sigma}_{\alpha \beta}^{\mu} \sigma_{\gamma \delta}^{\mu}=\sigma_{\alpha \beta}^{\mu} \bar{\sigma}_{\gamma \delta}^{\mu}=2 \delta_{\alpha \delta} \delta_{\beta \gamma}, \\
& \sigma_{\alpha \beta}^{\mu} \sigma_{\gamma \delta}^{\mu}=\bar{\sigma}_{\alpha \beta}^{\mu} \bar{\sigma}_{\gamma \delta}^{\mu}=2 \varepsilon_{\alpha \gamma} \varepsilon_{\beta \delta},
\end{aligned}
$$

where $\alpha, \beta, \gamma, \delta=1,2$ are quaternionic indices. The $\sigma^{\mu}$ and $\bar{\sigma}^{\mu}$ can be used to construct the Euclidean Dirac matrices as

$$
\begin{gathered}
\gamma^{\mu}=\left(\begin{array}{cc}
0 & \bar{\sigma}^{\mu} \\
\sigma^{\mu} & 0
\end{array}\right), \quad \gamma_{5}=\gamma_{1} \gamma_{2} \gamma_{3} \gamma_{4}=\left(\begin{array}{cc}
1 & 0 \\
0 & -1
\end{array}\right) \\
\left\{\gamma^{\mu}, \gamma^{\nu}\right\}=2 \delta^{\mu \nu}, \quad \gamma^{\mu \nu}=\frac{1}{2 i}\left[\gamma^{\mu}, \gamma^{\nu}\right]=\left(\begin{array}{cc}
\bar{\sigma}^{\mu \nu} & 0 \\
0 & \sigma^{\mu \nu}
\end{array}\right) .
\end{gathered}
$$

Thus (3.2) and (3.4) show that

$$
* \gamma^{\mu \nu} \frac{1 \pm \gamma_{5}}{2}=\mp \gamma^{\mu \nu} \frac{1 \pm \gamma_{5}}{2}
$$

We shall consider the propagator for spinor fields transforming in an arbitrary representation (fundamental, adjoint, etc.) of $U(N)$ gauge group in the background of (anti-)self-dual instantons. The covariant derivative $D_{\mu}$ is defined by

$$
D_{\mu}=\partial_{\mu}+A_{\mu}
$$


and the field strength $F_{\mu \nu}$ is given by

$$
\begin{aligned}
F_{\mu \nu} & =\left[D_{\mu}, D_{\nu}\right] \\
& =\partial_{\mu} A_{\nu}-\partial_{\nu} A_{\mu}+\left[A_{\mu}, A_{\nu}\right] .
\end{aligned}
$$

Since we are interested in spinor fields propagating in the background of (anti-)self-dual instantons, we will assume that the field strength satisfies the (anti-)self-duality condition

$$
F_{\mu \nu}= \pm * F_{\mu \nu}= \pm \frac{1}{2} \varepsilon_{\mu \nu \rho \sigma} F_{\rho \sigma} .
$$

Then we have

$$
(\gamma \cdot D)^{2} \frac{1 \pm \gamma_{5}}{2}=D^{2} \frac{1 \pm \gamma_{5}}{2}+\frac{i}{2} F^{\mu \nu} \gamma^{\mu \nu} \frac{1 \pm \gamma_{5}}{2}
$$

The equation (3.5) forces the second term of the right-hand side of (3.9) to vanish for spinors with positive (negative) chirality in the self-dual (anti-self-dual) instanton background. In this case there is no zero mode solution satisfying

$$
\gamma^{\mu} D_{\mu} \psi_{ \pm}^{(0)}=0, \quad \gamma_{5} \psi_{ \pm}^{(0)}= \pm \psi_{ \pm}^{(0)}
$$

However the second term in (3.9) does not vanish for positive (negative) chirality spinor in the anti-self-dual (self-dual) instantons. In this case a finite number of zero modes satisfying (3.10) can be found. In the background of $k$ instantons in $U(N)$ gauge theory, the number of zero modes is $k$ in the fundamental representation and $2 N k$ in the adjoint representation [42].

We will now consider a spinor field in the background of $k$ anti-self-dual instantons. The self-dual case is obtained simply by changing the sign of $\gamma_{5}, \gamma_{5} \rightarrow-\gamma_{5}$. Let's introduce eigenfunctions $\psi_{r}(x)$ such that

$$
\gamma^{\mu} D_{\mu} \psi_{r}(x)=\lambda_{r} \psi_{r}(x)
$$

to define the spin- $\frac{1}{2}$ Green function $S(x, y)$ which is described by the formal expression

$$
S(x, y)=\sum_{r}^{\prime} \lambda_{r}^{-1} \psi_{r}(x) \psi_{r}(y)^{\dagger}
$$

where the prime means that the zero modes (states with $\lambda_{r}=0$ ) are excluded from the sum. It follows from (3.12) that the spin- $\frac{1}{2}$ propagator is orthogonal to all the zero modes in (3.10)

$$
\operatorname{Tr}_{\mathcal{H}}^{x}\left(\psi_{n}^{(0)}(x)^{\dagger} S(x, y)\right)=0
$$

Thus the spin- $\frac{1}{2}$ propagator obeys the following equation

$$
\gamma^{\mu} D_{\mu} S(x, y)=Q(x, y)
$$

where

$$
Q(x, y)=\delta(x, y)-\sum_{n} \psi_{n}^{(0)}(x) \psi_{n}^{(0)}(y)^{\dagger}
$$


with the summation running over all the zero modes $(n=1, \cdots, k$ for spinors in the fundamental representation and $n=1, \cdots, 2 N k$ in the adjoint representation). The quantity $Q(x, y)$ represents the projection operator, i.e. $\operatorname{Tr}_{\mathcal{H}}^{z}(Q(x, z) Q(z, y))=Q(x, y)$, into the subspace of all nonzero modes.

Using the same operator technique as in 44, the construction of $S(x, y)$ can be easily achieved. Let's introduce an operator $S$ whose matrix representation with regard to position eigenstates in $\mathcal{H}_{p}$ is $S(x, y)$,

$$
\langle x|S| y\rangle=S(x, y)
$$

Similarly, we write the corresponding spin-0 propagator $G(x, y)$, which is defined by

$$
-D_{\mu} D_{\mu} G(x, y)=\delta(x-y)
$$

as the matrix element of an operator $\frac{1}{-D^{2}}$,

$$
\left\langle x\left|\frac{1}{-D^{2}}\right| y\right\rangle=G(x, y) .
$$

We will show that the operator expression of the spin- $\frac{1}{2}$ propagator is

$$
S=-\gamma \cdot D \frac{1}{-D^{2}} \frac{1-\gamma_{5}}{2}-\frac{1}{-D^{2}} \gamma \cdot D \frac{1+\gamma_{5}}{2}
$$

First note that

$$
\gamma \cdot D S=Q
$$

where

$$
Q=\frac{1-\gamma_{5}}{2}-\gamma \cdot D \frac{1}{-D^{2}} \gamma \cdot D \frac{1+\gamma_{5}}{2}
$$

The equation (3.20) implies that $Q$ contains no zero modes since they are annihilated by $\gamma \cdot D$. On the other hand, we find that

$$
\gamma \cdot D Q=\gamma \cdot D
$$

and

$$
Q S=S .
$$

It is easy to show that $Q^{2}=Q$. Therefore Eq.(3.22) shows that $Q$ is the operator which projects into the subspace of all nonzero modes and so $\langle x|Q| y\rangle$ is the function defined in (3.15). Moreover, Eq.(3.23) implies that $S$ is orthogonal to all the zero modes. This ensures our claim in (3.19).

Let's consider Yang-Mills theory with gauge group $U(N)$ with action

$$
S=-\frac{1}{2} \operatorname{Tr}{ }_{\mathcal{H}} \operatorname{tr}\left(F_{\mu \nu} F_{\mu \nu}+\frac{1}{\xi}\left(D_{\mu} A_{\mu}\right)^{2}\right)
$$

and small fluctuations about a classical instanton solution $A_{\mu}(x)$

$$
A_{\mu}^{\prime}(x)=A_{\mu}(x)+\delta A_{\mu}(x)
$$


If the action is expanded to second order in $\delta A_{\mu}$, one can find the following result

$$
S\left[A_{\mu}^{\prime}\right] \approx S\left[A_{\mu}\right]-\operatorname{Tr} \mathcal{H}_{\operatorname{tr}} \delta A_{\mu}\left(-D^{2} \delta_{\mu \nu}-2 F_{\mu \nu}+\left(1-\frac{1}{\xi}\right) D_{\mu} D_{\nu}\right) \delta A_{\nu}
$$

In our previous paper 42 we showed that in $k$ instanton background there are $4 N k$ adjoint zero modes $\phi_{\mu}^{(n)}, n=1, \cdots, 4 N k$ satisfying $D_{\mu} \phi_{\mu}^{(n)}=0$ and

$$
\left(D^{2} \delta_{\mu \nu}+2 F_{\mu \nu}\right) \phi_{\nu}^{(n)}=0
$$

Thus to define spin-1 propagator we should project out the zero modes just as the spin- $\frac{1}{2}$ propagator (3.14). According to the action (3.26), the spin-1 propagator $G_{\mu \nu}(x, y)$ in the antiself-dual $k$ instanton background is defined by

$$
\left(-D^{2} \delta_{\mu \lambda}-2 F_{\mu \lambda}+\left(1-\frac{1}{\xi}\right) D_{\mu} D_{\lambda}\right) G_{\lambda \nu}(x, y)=Q_{\mu \nu}(x, y)
$$

where

$$
Q_{\mu \nu}(x, y)=\delta_{\mu \nu} \delta(x-y)-\sum_{n} \phi_{\mu}^{(n)}(x) \phi_{\nu}^{(n)}(y)^{\dagger} .
$$

The quantity $Q_{\mu \nu}(x, y)$ is the projection operator, i.e. $\operatorname{Tr}_{\mathcal{H}}^{z} Q_{\mu \lambda}(x, z) Q_{\lambda \nu}(z, y)=Q_{\mu \nu}(x, y)$, onto the space of the nonzero modes.

Using the operator formalism used in the spin- $\frac{1}{2}$ propagator, one can show that the spin-1 propagator can also be constructed in terms of corresponding scalar propagator. To proceed with the construction, define

$$
q_{\mu \nu \lambda \kappa}^{( \pm)}=\delta_{\mu \nu} \delta_{\lambda \kappa}+\eta_{\mu \nu}^{( \pm) a} \eta_{\lambda \kappa}^{( \pm) a}=\delta_{\mu \lambda} \delta_{\nu \kappa}+\eta_{\mu \lambda}^{(\mp) a} \eta_{\nu \kappa}^{(\mp) a}
$$

where $\eta_{\mu \nu}^{(+) a}=\eta_{\mu \nu}^{a}$ and $\eta_{\mu \nu}^{(-) a}=\bar{\eta}_{\mu \nu}^{a}$ defined in (3.2). The tensor

$$
\eta_{\mu \nu}^{( \pm) a} \eta_{\lambda \kappa}^{( \pm) a}=\frac{1}{4}\left(\delta_{\mu \lambda} \delta_{\nu \kappa}-\delta_{\mu \kappa} \delta_{\nu \lambda} \pm \varepsilon_{\mu \nu \lambda \kappa}\right)
$$

projects out the self-dual part or anti-self-dual part of an antisymmetric tensor since $\eta_{\mu \nu}^{( \pm) a} \eta_{\mu \nu}^{(\mp) b}=$ 0. Following [44, let's introduce a bracket operation

$$
\{X\}_{\mu \nu}^{( \pm)}=q_{\mu \nu \lambda \kappa}^{( \pm)} D_{\lambda} X D_{\kappa}
$$

for an arbitrary operator $X$. Then it is easy to see that

$$
D_{\nu}\{X\}_{\nu \mu}^{( \pm)}=D^{2} X D_{\mu}+\left[F_{\mu \nu} \mp * F_{\mu \nu}, X D_{\nu}\right]
$$

So if the field strength satisfies the self-duality condition (3.8), Eq.(3.33) reduces to

$$
D_{\nu}\{X\}_{\nu \mu}^{( \pm)}=D^{2} X D_{\mu}
$$


Similarly,

$$
\{X\}_{\mu \nu}^{( \pm)} D_{\nu}=D_{\mu} D^{2} X
$$

Let's quote the following algebraic relation 444]

$$
q_{\mu \lambda \nu \sigma}^{( \pm)} q_{\lambda \kappa \tau \rho}^{( \pm)}=\delta_{\sigma \tau} q_{\mu \kappa \nu \rho}^{( \pm)}+r_{\mu \kappa \nu \rho \sigma \tau}^{( \pm)}
$$

where

$$
r_{\mu \kappa \nu \rho \sigma \tau}^{( \pm)}=\left(\delta_{\mu \nu} \eta_{\kappa \rho}^{(\mp) c}-\delta_{\kappa \rho} \eta_{\mu \nu}^{(\mp) c}+\varepsilon_{a b c} \eta_{\mu \nu}^{(\mp) a} \eta_{\kappa \rho}^{(\mp) b}\right) \eta_{\sigma \tau}^{(\mp) c}
$$

and thus it has the following duality property

$$
\frac{1}{2} \varepsilon_{\sigma \tau v \lambda} r_{\mu \kappa \nu \rho \sigma \tau}^{( \pm)}=\mp r_{\mu \kappa \nu \rho v \lambda}^{( \pm)} .
$$

In the derivation of (3.37), we used

$$
\eta_{\lambda \mu}^{( \pm) a} \eta_{\lambda \nu}^{( \pm) b}=\delta_{a b} \delta_{\mu \nu}+\varepsilon_{a b c} \eta_{\mu \nu}^{( \pm) c} .
$$

Using these properties, the following bracket composition law can be derived

$$
\{X\}_{\mu \lambda}^{( \pm)}\{Y\}_{\lambda \nu}^{( \pm)}=\left\{X D^{2} Y\right\}_{\mu \nu}^{( \pm)} .
$$

Now it is straightforward to see that $G_{\mu \nu}(x, y)$ has the following formal operator expression

$$
G_{\mu \nu}=-\left\{\left(\frac{1}{D^{2}}\right)^{2}\right\}_{\mu \nu}+(1-\xi) D_{\mu}\left(\frac{1}{D^{2}}\right)^{2} D_{\nu} .
$$

The reason is following. First note that

$$
\left(-D^{2} \delta_{\mu \lambda}-2 F_{\mu \lambda}+\left(1-\frac{1}{\xi}\right) D_{\mu} D_{\lambda}\right) G_{\lambda \nu}=Q_{\mu \nu}
$$

where

$$
Q_{\mu \nu}=\left\{\frac{1}{D^{2}}\right\}_{\mu \nu} .
$$

We used (3.34) and (3.35) and the bracket composition (3.40) in the derivation. Comparing with (3.28), we see that $Q_{\mu \nu}$ doesn't contain any zero modes. And, using the composition law (3.40), one can easily see that $Q_{\mu \nu}$ is a projection operator, i.e. $Q_{\mu \lambda} Q_{\lambda \nu}=Q_{\mu \nu}$. Indeed, $Q_{\mu \nu}$ is the projection operator onto all the nonzero modes in (3.29) and thus an operator realization of the projector $Q_{\mu \nu}(x, y)$ since it satisfies the following equations

$$
\begin{aligned}
& Q_{\mu \lambda} G_{\lambda \nu}=G_{\mu \nu}, \\
& \left(-D^{2} \delta_{\mu \lambda}-2 F_{\mu \lambda}+\left(1-\frac{1}{\xi}\right) D_{\mu} D_{\lambda}\right) Q_{\lambda \nu}=\left(-D^{2} \delta_{\mu \nu}-2 F_{\mu \nu}+\left(1-\frac{1}{\xi}\right) D_{\mu} D_{\nu}\right) .
\end{aligned}
$$

Thus we complete the proof of our claim in (3.41). 


\section{Scalar Instanton Propagators}

In order to calculate instanton effects in quantum gauge theory, it is important to know the Green function in instanton backgrounds [6]. In previous section, following the same method in [44, we showed that the propagators for spinor and vector fields can be constructed in terms of those for the scalar field in noncommutative instanton background. Thus, if we can find the scalar propagator $G(x, y)$ (3.17) for fundamental representation or adjoint representation, we know the spin- $\frac{1}{2}$ propagator $S(x, y)$ for each representation in terms of (3.19) and the spin-1 propagator $G_{\mu \nu}(x, y)$ in terms of (3.41). In commutative space, the scalar propagator in the fundamental representation has a remarkably simple expression [50, 51]

$$
G(x, y)=v(x)^{\dagger} G^{(0)}(x, y) v(y)
$$

where $v(x)$ is a function determining ADHM gauge field $A_{\mu}(x)$ by $A_{\mu}(x)=v(x)^{\dagger} \partial_{\mu} v(x)$. The scalar propagator in the adjoint representation has more complicated expression of which we will present the explicit form. We will first show that the scalar propagator in the noncommutative instanton background has the exactly same form that (4.1).

To derive the above remarkable formulae, we need the following basic properties in the ADHM construction [18, 50, 51]. The gauge field with instanton number $k$ for $U(N)$ gauge group is given in the form

$$
A_{\mu}(x)=v(x)^{\dagger} \partial_{\mu} v(x)
$$

where $v(x)$ is $(N+2 k) \times N$ matrix defined by the equations

$$
\begin{aligned}
& v(x)^{\dagger} v(x)=1, \\
& v(x)^{\dagger} \Delta(x)=0 .
\end{aligned}
$$

In (4.4), $\Delta(x)$ is a $(N+2 k) \times 2 k$ matrix, linear in the position variable $x$, having the structure

$$
\Delta(x)= \begin{cases}a-b \mathbf{x}, & \text { self-dual instantons, } \\ a-b \overline{\mathbf{x}}, & \text { anti-self-dual instantons }\end{cases}
$$

where $a, b$ are $(N+2 k) \times 2 k$ matrices. $v(x)$ can be thought of as a map from an $N$-complex dimensional space $W$ to a $N+2 k$-complex dimensional space $V$. Thus $\Delta(x)$ must obey the completeness relation

$$
P(x)+\Delta(x) f(x) \Delta(x)^{\dagger}=1
$$

where $P(x)=v(x) v(x)^{\dagger}$. The matrices $a, b$ are constrained to satisfy the conditions that $\Delta(x)^{\dagger} \Delta(x)$ be invertible and that it commutes with the quaternions. These conditions imply that $\Delta(x)^{\dagger} \Delta(x)$ as a $2 k \times 2 k$ matrix has to be factorized as follows

$$
\Delta(x)^{\dagger} \Delta(x)=f^{-1}(x) \otimes 1_{2}
$$


where $f^{-1}(x)$ is a $k \times k$ matrix and $1_{2}$ is a unit matrix in quaternion space.

Given a pair of matrices $a, b,(4.3)$ and (4.4) define $A_{\mu}$ up to gauge equivalence. Different pair of matrices $a, b$ may yield gauge equivalent $A_{\mu}$ since (4.3) and (4.4) are invariant under

$$
a \rightarrow Q a K, \quad b \rightarrow Q b K, \quad v \rightarrow Q v
$$

where $Q \in U(N+2 k)$ and $K \in G L(k, \mathbf{C})$. This freedom can be used to put $a, b$ in the canonical forms

$$
a=\left(\begin{array}{c}
\lambda \\
\xi
\end{array}\right), \quad b=\left(\begin{array}{c}
0 \\
1_{2 k}
\end{array}\right),
$$

where $\lambda$ is an $N \times 2 k$ matrix and $\xi$ is a $2 k \times 2 k$ matrix. Here we decompose the matrix $\xi$ in the quaternionic basis $\bar{\sigma}^{\mu}$ as a matter of convenience

$$
\xi=\xi_{\mu} \bar{\sigma}^{\mu}
$$

where $\xi_{\mu}$ 's are $k \times k$ matrices. In the basis (4.9), the constraint (4.7) boils down to

$$
\begin{aligned}
& \operatorname{tr}_{2} \tau^{a} a^{\dagger} a= \begin{cases}\theta^{\mu \nu} \bar{\eta}_{\mu \nu}^{a}, & \text { self-dual instantons }, \\
\theta^{\mu \nu} \eta_{\mu \nu}^{a}, & \text { anti-self-dual instantons }\end{cases} \\
& \xi_{\mu}^{\dagger}=\xi_{\mu},
\end{aligned}
$$

where $\operatorname{tr}_{2}$ is the trace over the quaternionic indices.

\subsection{Scalar Propagator in Fundamental Representation}

Now we will explain how to derive the formulae (4.1). First note that the covariant derivative for a field $\Phi$ in the fundamental representation of $U(N)$ has the simple expression

$$
D_{\mu} \Phi=\left(\partial_{\mu}+v^{\dagger} \partial_{\mu} v\right) \Phi=v^{\dagger} \partial_{\mu}(v \Phi)
$$

Using this relation, let's calculate $-D_{\mu} D_{\mu} G(x, y)$,

$$
\begin{aligned}
& -D_{\mu} D_{\mu}\left(v(x)^{\dagger} G^{(0)}(x, y) v(y)\right) \\
= & -v(x)^{\dagger} \partial_{\mu}\left(P(x) \partial_{\mu}\left(P(x) G^{(0)}(x, y) v(y)\right)\right) .
\end{aligned}
$$

Note that $v(x)^{\dagger} P(x)=v(x)^{\dagger}$, so

$$
\begin{aligned}
& -D_{\mu} D_{\mu} G(x, y)=-v(x)^{\dagger} \partial_{\mu}\left(P(x) \partial_{\mu} P(x)\right) G^{(0)}(x, y) v(y) \\
& -2 v(x)^{\dagger}\left(\partial_{\mu} P(x) \partial_{\mu} G^{(0)}(x, y)\right) v(y)-v(x)^{\dagger} \partial_{\mu} \partial_{\mu} G^{(0)}(x, y) v(y) .
\end{aligned}
$$


Let's calculate the first term of the right-hand side in (4.15)

$$
\begin{aligned}
& v(x)^{\dagger} \partial_{\mu}\left(P(x) \partial_{\mu} P(x)\right) \\
= & -v(x)^{\dagger} \partial_{\mu}\left(P(x) \partial_{\mu} \Delta(x) f(x) \Delta(x)^{\dagger}\right) \\
= & v(x)^{\dagger} \partial_{\mu} \Delta(x) f(x) \Delta(x)^{\dagger} \partial_{\mu} \Delta(x) f(x) \Delta(x)^{\dagger}-v(x)^{\dagger} \partial_{\mu} \Delta(x) \partial_{\mu} f(x) \Delta(x)^{\dagger} \\
& -v(x)^{\dagger} \partial_{\mu} \Delta(x) f(x) \partial_{\mu} \Delta(x)^{\dagger}
\end{aligned}
$$

where we used (4.4) and (4.6). Also note that

$$
\partial_{\mu} f(x)=-f(x)\left(\partial_{\mu} \Delta(x)^{\dagger} \Delta(x)+\Delta(x)^{\dagger} \partial_{\mu} \Delta(x)\right) f(x)
$$

from (4.7).

For explicit calculation, let's take the anti-self-dual instanton with $\Delta(x)=a-b \overline{\mathbf{x}}$. The self-dual case can be similarly calculated. Using $\partial_{\mu} \Delta(x)=-b \bar{\sigma}_{\mu}$ and $\partial_{\mu} \Delta(x)^{\dagger}=-\sigma_{\mu} b^{\dagger}$ and the following formula

$$
\bar{\sigma}_{\mu} \Delta(x)^{\dagger} b \bar{\sigma}_{\mu}=-2 b^{\dagger} \Delta(x), \quad \bar{\sigma}_{\mu} f(x) \sigma_{\mu}=4 f(x),
$$

we arrive at

$$
v(x)^{\dagger} \partial_{\mu}\left(P(x) \partial_{\mu} P(x)\right)=-4 v(x)^{\dagger} b f(x) b^{\dagger},
$$

where we used the fact that the function $f(x)$ commutes with $\bar{\sigma}_{\mu}$ and $\sigma_{\mu}$. Then our original equation (4.15) reduces to

$$
-D_{\mu} D_{\mu} G(x, y)=2 v(x)^{\dagger}\left(2 b f(x) b^{\dagger} G^{(0)}(x, y)-\partial_{\mu} P(x) \partial_{\mu} G^{(0)}(x, y)\right) v(y)+\delta(x-y)
$$

where $-\partial_{\mu} \partial_{\mu} G^{(0)}(x, y)=\delta(x-y)$ is used. Note that the whole procedure above until (4.20) is totally valid even for noncommutative space.

To arrive at our final destination (3.17), we must show that

$$
v(x)^{\dagger}\left(2 b f(x) b^{\dagger} G^{(0)}(x, y)-\partial_{\mu} P(x) \partial_{\mu} G^{(0)}(x, y)\right) v(y)=0 .
$$

First let's show (4.21) in commutative $\mathbf{R}^{4}$, where we don't have to worry about the ordering problem, which will also be helpful to find the noncommutative version. If one notices that $\Delta(y)^{\dagger} v(y)=0$ and

$$
\partial_{\mu} G^{(0)}(x, y)=-G^{(0)}(x, y) \frac{2(x-y)_{\mu}}{(x-y)^{2}},
$$

the second term of (4.21) can be written as

$$
\begin{aligned}
& v(x)^{\dagger} \partial_{\mu} P(x) \partial_{\mu} G^{(0)}(x, y) v(y) \\
= & v(x)^{\dagger} b \bar{\sigma}_{\mu} f(x) \partial_{\mu} G^{(0)}(x, y)\left(\Delta(x)^{\dagger}-\Delta(y)^{\dagger}\right) v(y) \\
= & 2 v(x)^{\dagger} b f(x) \bar{\sigma}_{\mu} \sigma_{\nu} b^{\dagger} G^{(0)}(x, y) \frac{(x-y)_{\mu}}{(x-y)^{2}}(x-y)_{\nu} v(y) .
\end{aligned}
$$


Since $\bar{\sigma}_{\mu} \sigma_{\nu}=\delta_{\mu \nu}+i \bar{\sigma}_{\mu \nu}$, (4.23) exactly cancels the first term in (4.21). Thus we proved (4.1) in the commutative $\mathbf{R}^{4}$.

Before going on a tour to noncommutative space, let's explain why we expect (4.1) even for the noncommutative space. The relation (4.13) implies that if we define

$$
\hat{\Phi}=v \Phi \quad \text { and } \quad D_{\mu} \hat{\Phi}=\widehat{D_{\mu} \Phi}
$$

we get

$$
D_{\mu} \hat{\Phi}=P \partial_{\mu} \hat{\Phi}
$$

We may interpret this result as follows [50]. The matrix $v: W \rightarrow V$ maps $\Phi$ in the $N$ dimensional complex vector space $W$ with $\hat{\Phi}$ in an $N+2 k$-dimensional complex vector space $V$ which lies in an $N$-dimensional subspace of $\mathbf{C}^{N+2 k}$, i.e. the subspace

$$
E_{x}=\{\xi \mid P(x) \xi=\xi\}
$$

orthogonal to $\Delta(x)$ onto which $P$ is the projection operator. The collection of spaces $\left\{E_{x}\right\}$ as $x$ varies over $\mathbf{R}^{4}$ or $\mathbf{R}_{N C}^{4}$ forms a vector bundle and this vector bundle precisely defines the ADHM gauge fields $A_{\mu}(x)$ through (4.25). This is the statement of Serre-Swan theorem [52]; the vector bundle over a $C^{*}$-algebra $\mathcal{A}$ (which is a complex Banach algebra with adjoint operation) is a finitely generated projective module. (A module $\mathcal{E}$ is projective if there exists another module $\mathcal{F}$ such that the direct sum $\mathcal{E} \oplus \mathcal{F}$ is free, i.e. $\mathcal{E} \oplus \mathcal{F} \cong \mathcal{A} \otimes \cdots \otimes \mathcal{A}$ as right $\mathcal{A}$-module.) Thus one can imagine the Green function $G(x, y)$ for the field $\Phi$ living in the "nontrivial" $N$-dimensional vector space $W$, which is defined as $G(x, y)=\langle\Phi(x), \Phi(y)\rangle$, is obtained by the map $v: W \rightarrow V$ from the Green function $G^{(0)}(x, y) \equiv\langle\hat{\Phi}(x), \hat{\Phi}(y)\rangle$ for the field $\hat{\Phi}$ living in the "free" $N+2 k$-dimensional vector space $V$. This is precisely the content of (4.1). Note, however, that this argument should also be valid for a noncommutative space. This is the reason why we expect the propagator (4.1) even for noncommutative instanton background.

To show (4.21) in the noncommutative space (2.1), let's chase the previous commutative calculation keeping in mind the ordering due to the noncommutativity. The second term in 4.21) can be written as

$$
\begin{aligned}
& v(x)^{\dagger} \partial_{\mu} P(x) \partial_{\mu} G^{(0)}(x, y) v(y) \\
= & v(x)^{\dagger} b \bar{\sigma}_{\mu} f(x) \Delta(x)^{\dagger} \partial_{\mu} G^{(0)}(x, y) v(y) \\
= & v(x)^{\dagger} b f(x) \bar{\sigma}_{\mu} \partial_{\mu} G^{(0)}(x, y)\left(\Delta(x)^{\dagger}-\Delta(y)^{\dagger}\right) v(y)+v(x)^{\dagger} b f(x) \bar{\sigma}_{\mu}\left[\Delta(x)^{\dagger}, \partial_{\mu} G^{(0)}(x, y)\right] v(y) \\
= & -v(x)^{\dagger} b f(x) \bar{\sigma}_{\mu} \sigma_{\nu} b^{\dagger} \partial_{\mu} G^{(0)}(x, y)(x-y)_{\nu} v(y)-v(x)^{\dagger} b f(x) \bar{\sigma}_{\mu} \sigma_{\nu} b^{\dagger}\left[x_{\nu}, \partial_{\mu} G^{(0)}(x, y)\right] v(y) \\
= & -v(x)^{\dagger} b f(x) \bar{\sigma}_{\mu} \sigma_{\nu} b^{\dagger}(x-y)_{\nu} \partial_{\mu} G^{(0)}(x, y) v(y)-v(x)^{\dagger} b f(x) \bar{\sigma}_{\mu} \sigma_{\nu} b^{\dagger}\left[y_{\nu}, \partial_{\mu} G^{(0)}(x, y)\right] v(y) \\
= & -\frac{1}{2} v(x)^{\dagger} b f(x) \bar{\sigma}_{\mu} \sigma_{\nu} b^{\dagger}\left(\partial_{\mu} G^{(0)}(x, y)(x-y)_{\nu}+(x-y)_{\nu} \partial_{\mu} G^{(0)}(x, y)\right) v(y)
\end{aligned}
$$




$$
\begin{aligned}
& -\frac{1}{2} v(x)^{\dagger} b f(x) \bar{\sigma}_{\mu} \sigma_{\nu} b^{\dagger}\left[(x+y)_{\nu}, \partial_{\mu} G^{(0)}(x, y)\right] v(y) \\
= & -\frac{1}{2} v(x)^{\dagger} b f(x) \bar{\sigma}_{\mu} \sigma_{\nu} b^{\dagger}\left(\partial_{\mu} G^{(0)}(x, y)(x-y)_{\nu}+(x-y)_{\nu} \partial_{\mu} G^{(0)}(x, y)\right) v(y) .
\end{aligned}
$$

In the last step, we used the fact that the function $\partial_{\mu} G^{(0)}(x, y)$ depends only on the combination $(x-y)$ because of translation invariance and (2.26).

In order to calculate the right-hand side of (4.27), we will use the Weyl symmetric prescription (2.18):

$$
\begin{aligned}
& \frac{1}{2} \bar{\sigma}_{\mu} \sigma_{\nu}\left(\partial_{\mu} G^{(0)}(x, y)(x-y)_{\nu}+(x-y)_{\nu} \partial_{\mu} G^{(0)}(x, y)\right) \\
= & \int \frac{d^{4} k}{(2 \pi)^{4}} \bar{\sigma}_{\mu} \sigma_{\nu} \frac{k_{\mu}}{k^{2}} \frac{\partial}{\partial k^{\nu}} e^{i k \cdot(x-y)} \\
= & \int \frac{d^{4} k}{(2 \pi)^{4}} \bar{\sigma}_{\mu} \sigma_{\nu} \frac{\partial}{\partial k^{\nu}}\left(\frac{k_{\mu}}{k^{2}} e^{i k \cdot(x-y)}\right)-\int \frac{d^{4} k}{(2 \pi)^{4}} \bar{\sigma}_{\mu} \sigma_{\nu} \frac{1}{k^{2}}\left(\delta_{\mu \nu}-2 \frac{k_{\mu} k_{\nu}}{k^{2}}\right) e^{i k \cdot(x-y)} \\
= & \int \frac{d^{4} k}{(2 \pi)^{4}} \bar{\sigma}_{\mu} \sigma_{\nu} \frac{\partial}{\partial k^{\nu}}\left(\frac{k_{\mu}}{k^{2}} e^{i k \cdot(x-y)}\right)-2 G^{(0)}(x, y) .
\end{aligned}
$$

Thus if we can show that the total divergence $\int \frac{d^{4} k}{(2 \pi)^{4}} \bar{\sigma}_{\mu} \sigma_{\nu} \frac{\partial}{\partial k^{\nu}}\left(\frac{k_{\mu}}{k^{2}} e^{i k \cdot(x-y)}\right) \equiv K(x-y)$ vanishes on $\mathbf{S}^{3}$ in the large $k$ limit, we can finally achieve our goal (4.21) in the noncommutative space. It is easy to show directly in the basis of tensor product $\left|\xi_{1}, \xi_{2}\right\rangle=\left|\xi_{1}\right\rangle \otimes\left|\xi_{2}\right\rangle$ of coherent states such as (2.14), using (2.17), that the function $\left\langle\xi_{1}, \xi_{2}|K(x-y)| \xi_{1}, \xi_{2}\right\rangle$ vanishes for any $\left|\xi_{1}, \xi_{2}\right\rangle$. This means that the operator function $K(x-y)$ should vanish even in the noncommutative space.

\subsection{Scalar Propagator in Adjoint Representation}

Next let's consider the scalar propagator in the adjoint representation. If $q$ denotes the fundamental representation of $U(N)$, the adjoint representation can be obtained by tensor product $q \otimes \bar{q}$, for which

$$
D_{\mu}=\partial_{\mu}+A_{\mu} \otimes 1+1 \otimes \bar{A}_{\mu} .
$$

In other word, we regard a field in the adjoint representation as a two index object, one index transforming according to the fundamental representation and the other its complex conjugate. Motivated by this fact and to follow the method in [45], we treat this problem in a more general context. Consider the direct product $G_{1} \times G_{2}$ and suppose we have instanton solutions

$$
A_{\mu}^{1}(x)=v_{1}(x)^{\dagger} \partial_{\mu} v_{1}(x), \quad A_{\mu}^{2}(x)=v_{2}(x)^{\dagger} \partial_{\mu} v_{2}(x),
$$

described in the ADHM way for each gauge group. Also consider a field transforming under the fundamental representation of each and thus its covariant derivative is defined by

$$
D_{\mu}=\partial_{\mu}+A_{\mu}^{1} \otimes 1+1 \otimes A_{\mu}^{2}
$$


The adjoint representation of $U(N)$ would be obtained by taking $G_{1}=G_{2}=U(N)$ and $A_{\mu}^{1}=A_{\mu}^{2}=A_{\mu}$.

The Green function for a tensor product should be obtained by solving (3.17) with $D_{\mu}$ defined by (4.31). Thus we will also consider the tensor product

$$
v(x)=v_{1}(x) \otimes v_{2}(x)
$$

of two independent fields $v_{1}(x), v_{2}(x)$ in the fundamental representation of $G_{1}$ and $G_{2}$ respectively. To preserve the group structure for $G_{1} \times G_{2}$, i.e. $\left(g_{1}, g_{2}\right)\left(h_{1}, h_{2}\right)=\left(g_{1} h_{1}, g_{2} h_{2}\right) \in G_{1} \times G_{2}$ for all $g_{1}, h_{1} \in G_{1}$ and $g_{2}, h_{2} \in G_{2}$, we define a (unique) multiplication between elements of $G_{1} \times G_{2}$ such that

$$
\left(\phi_{1}(x) \otimes \phi_{2}(x)\right)\left(\chi_{1}(x) \otimes \chi_{2}(x)\right)=\left(\phi_{1}(x) \chi_{1}(x)\right) \otimes\left(\phi_{2}(x) \chi_{2}(x)\right)
$$

for all $\phi_{1}(x), \chi_{1}(x) \in G_{1}$ and $\phi_{2}(x), \chi_{2}(x) \in G_{2}$. This multiplication law will be crucial for our calculation of adjoint Green function. The commutative Green function $G(x, y)$ satisfying (3.17) for $G_{1} \times G_{2}$ was previously constructed in [45] and to be of the form

$$
G(x, y)=\left[v_{1}(x) \otimes v_{2}(x)\right]^{\dagger} G^{(0)}(x, y)\left[v_{1}(y) \otimes v_{2}(y)\right]+\frac{1}{4 \pi^{2}} C(x, y) .
$$

We will specify the explicit form of $C(x, y)$ later.

We will try the same expression as (4.34) to construct noncommutative instanton propagator. To calculate (3.17) for the ansatz (4.34), first note that, using the multiplication law $4.33)$

$$
\begin{aligned}
D_{\mu} v(x)^{\dagger}= & v_{1}(x)^{\dagger} \partial_{\mu} P_{1}(x) \otimes v_{2}(x)^{\dagger}+v_{1}(x)^{\dagger} \otimes v_{2}(x)^{\dagger} \partial_{\mu} P_{2}(x), \\
D_{\mu} D_{\mu} v(x)^{\dagger}= & v_{1}(x)^{\dagger} \partial_{\mu}\left(v_{1}(x)^{\dagger} \partial_{\mu} P_{1}(x)\right) \otimes v_{2}(x)^{\dagger}+v_{1}(x)^{\dagger} \otimes v_{2}(x)^{\dagger} \partial_{\mu}\left(v_{2}(x)^{\dagger} \partial_{\mu} P_{2}(x)\right) \\
& +2 v_{1}(x)^{\dagger} \partial_{\mu} P_{1}(x) \otimes v_{2}(x)^{\dagger} \partial_{\mu} P_{2}(x),
\end{aligned}
$$

where we defined

$$
P_{a}(x)=v_{a}(x) v_{a}(x)^{\dagger}, \quad a=1,2 .
$$

If we further define

$$
P(x)=P_{1}(x) \otimes P_{2}(x),
$$

the above covariant derivatives can be rewritten as, using (4.33) again,

$$
\begin{aligned}
D_{\mu} v(x)^{\dagger} & =v(x)^{\dagger} \partial_{\mu} P(x), \\
D_{\mu} D_{\mu} v(x)^{\dagger} & =v(x)^{\dagger} \partial_{\mu}\left(P(x) \partial_{\mu} P(x)\right),
\end{aligned}
$$

where we used $v_{1}^{\dagger}(x) P_{1}(x)=v_{1}^{\dagger}(x)$ and $v_{2}^{\dagger}(x) P_{2}(x)=v_{2}^{\dagger}(x)$. Thus we can proceed with the calculation of $-D_{\mu} D_{\mu}\left(v(x)^{\dagger} G^{(0)}(x, y) v(y)\right)$ in the same way as Sec.3.1. Consequently we get

$$
\begin{aligned}
& -D_{\mu} D_{\mu}\left(v(x)^{\dagger} G^{(0)}(x, y) v(y)\right)=\delta(x-y) \\
& -2\left(v_{1}(x)^{\dagger} \partial_{\mu} P_{1}(x) \otimes v_{2}(x)^{\dagger} \partial_{\mu} P_{2}(x)\right) G^{(0)}(x, y)\left(v_{1}(y) \otimes v_{2}(y)\right) .
\end{aligned}
$$


Let's calculate the second term in (4.41). For explicit calculation, let's take the anti-self-dual instanton with $\Delta_{a}(x)=a_{a}-b_{a} \overline{\mathbf{x}}$. The self-dual case can be similarly done. Note that, according to ADHM construction, $v_{a}(x)^{\dagger} \partial_{\mu} P_{a}(x)=v_{a}(x)^{\dagger} b_{a} \bar{\sigma}_{\mu} f_{a}(x) \Delta_{a}(x)^{\dagger}$. Again the multiplication law (4.33) will have a crucial role in the calculation below,

$$
\begin{aligned}
& -2\left(v_{1}(x)^{\dagger} \partial_{\mu} P_{1}(x) \otimes v_{2}(x)^{\dagger} \partial_{\mu} P_{2}(x)\right) G^{(0)}(x, y) v(y) \\
= & -2\left(v_{1}(x)^{\dagger} b_{1} \bar{\sigma}_{\mu} f_{1}(x) \Delta_{1}(x)^{\dagger} \otimes v_{2}(x)^{\dagger} \partial_{\mu} P_{2}(x)\right) G^{(0)}(x, y) v(y) \\
= & -2\left(v_{1}(x)^{\dagger} b_{1} \bar{\sigma}_{\mu} f_{1}(x) \otimes v_{2}(x)^{\dagger} \partial_{\mu} P_{2}(x)\right) \times \\
& \left(\left[\Delta_{1}(x)^{\dagger} \otimes 1, G^{(0)}(x, y)\right]+G^{(0)}(x, y)\left(\Delta_{1}(x)^{\dagger}-\Delta_{1}(y)^{\dagger}\right) \otimes 1\right) v(y) \\
=\quad & -2\left(v_{1}(x)^{\dagger} b_{1} \bar{\sigma}_{\mu} f_{1}(x) \otimes v_{2}(x)^{\dagger} \partial_{\mu} P_{2}(x)\right) \times \\
& \left(\left[\sigma_{\nu} b_{1}^{\dagger} y_{\nu} \otimes 1, G^{(0)}(x, y)\right]+\left(\sigma_{\nu} b_{1}^{\dagger}(x-y)_{\nu} \otimes 1\right) G^{(0)}(x, y)\right) v(y) \\
= & -\left(v_{1}(x)^{\dagger} b_{1} \bar{\sigma}_{\mu} f_{1}(x) \otimes v_{2}(x)^{\dagger} \partial_{\mu} P_{2}(x)\right) \times \\
& \left(G^{(0)}(x, y)\left(\sigma_{\nu} b_{1}^{\dagger}(x-y)_{\nu} \otimes 1\right)+\left(\sigma_{\nu} b_{1}^{\dagger}(x-y)_{\nu} \otimes 1\right) G^{(0)}(x, y)\right) v(y) .
\end{aligned}
$$

In the last step, we used the fact that the function $\partial_{\mu} G^{(0)}(x, y)$ depends only on the combination $(x-y)$ and (2.26). We can repeat the same procedure in (4.42) for the term $v_{2}(x)^{\dagger} \partial_{\mu} P_{2}(x)$. Then we get

$$
\begin{aligned}
& -2\left(v_{1}(x)^{\dagger} \partial_{\mu} P_{1}(x) \otimes v_{2}(x)^{\dagger} \partial_{\mu} P_{2}(x)\right) G^{(0)}(x, y) v(y) \\
= & -\frac{1}{2}\left(v_{1}(x)^{\dagger} b_{1} \bar{\sigma}_{\mu} f_{1}(x) \otimes v_{2}(x)^{\dagger} b_{2} \bar{\sigma}_{\mu} f_{2}(x)\right) \times \\
& \left(F^{(0)}(x, y)\left(1 \otimes \sigma_{\lambda} b_{2}^{\dagger}(x-y)_{\lambda}\right)+\left(1 \otimes \sigma_{\lambda} b_{2}^{\dagger}(x-y)_{\lambda}\right) F^{(0)}(x, y)\right) v(y),
\end{aligned}
$$

where $F^{(0)}(x, y)=G^{(0)}(x, y)\left(\sigma_{\nu} b_{1}^{\dagger}(x-y)_{\nu} \otimes 1\right)+\left(\sigma_{\nu} b_{1}^{\dagger}(x-y)_{\nu} \otimes 1\right) G^{(0)}(x, y)$.

In order to calculate the right-hand side of (4.43), we will again use the Weyl symmetric prescription (2.18). First note that

$$
F^{(0)}(x, y)=-2 i\left(\sigma_{\nu} b_{1}^{\dagger} \otimes 1\right) \int \frac{d^{4} k}{(2 \pi)^{4}} \frac{1}{k^{2}} \frac{\partial}{\partial k^{\nu}} e^{i k \cdot(x-y)}
$$

and

$$
\begin{aligned}
& F^{(0)}(x, y)\left(1 \otimes \sigma_{\lambda} b_{2}^{\dagger}(x-y)_{\lambda}\right)+\left(1 \otimes \sigma_{\lambda} b_{2}^{\dagger}(x-y)_{\lambda}\right) F^{(0)}(x, y) \\
= & -4\left(\sigma_{\nu} b_{1}^{\dagger} \otimes \sigma_{\lambda} b_{2}^{\dagger}\right) \int \frac{d^{4} k}{(2 \pi)^{4}} \frac{1}{k^{2}} \frac{\partial^{2}}{\partial k^{\nu} \partial k^{\lambda}} e^{i k \cdot(x-y)} \\
\equiv & -4\left(\sigma_{\nu} b_{1}^{\dagger} \otimes \sigma_{\lambda} b_{2}^{\dagger}\right) H_{\nu \lambda}(x, y) .
\end{aligned}
$$


Then we arrive at

$$
\begin{aligned}
& -2\left(v_{1}(x)^{\dagger} \partial_{\mu} P_{1}(x) \otimes v_{2}(x)^{\dagger} \partial_{\mu} P_{2}(x)\right) G^{(0)}(x, y) v(y) \\
= & 2\left(v_{1}(x)^{\dagger} b_{1} \bar{\sigma}_{\mu} \sigma_{\nu} f_{1}(x) b_{1}^{\dagger} \otimes v_{2}(x)^{\dagger} b_{2} \bar{\sigma}_{\mu} \sigma_{\lambda} f_{2}(x) b_{2}^{\dagger}\right) H_{\nu \lambda}(x, y) v(y), \\
= & 2\left(v_{1}(x)^{\dagger} b_{1} \bar{\sigma}_{\mu} f_{1}(x) b_{1}^{\dagger} \otimes v_{2}(x)^{\dagger} b_{2} \bar{\sigma}_{\mu} f_{2}(x) b_{2}^{\dagger}\right) H_{\nu \nu}(x, y) v(y) .
\end{aligned}
$$

To derive the last result in (4.46), we used (3.2), (3.39) and the fact that $H_{\nu \lambda}(x, y)=H_{\lambda \nu}(x, y)$.

In order to calculate $H_{\nu \nu}(x, y)$, we introduce an infrared cutoff $\epsilon$, i.e. $\frac{1}{k^{2}} \rightarrow \frac{1}{k^{2}+\epsilon}$. After the integral, we will take the limit $\epsilon \rightarrow 0$ :

$$
\begin{aligned}
H_{\nu \nu}(x, y)= & \lim _{\epsilon \rightarrow 0} \int \frac{d^{4} k}{(2 \pi)^{4}} \frac{\partial}{\partial k_{\nu}}\left(\frac{1}{k^{2}+\epsilon} \frac{\partial}{\partial k^{\nu}} e^{i k \cdot(x-y)}+\frac{2 k^{\nu}}{\left(k^{2}+\epsilon\right)^{2}} e^{i k \cdot(x-y)}\right) \\
& -\lim _{\epsilon \rightarrow 0} \int \frac{d^{4} k}{(2 \pi)^{4}} \frac{8 \epsilon}{\left(k^{2}+\epsilon\right)^{3}} e^{i k \cdot(x-y)} .
\end{aligned}
$$

One can check that

$$
\lim _{\epsilon \rightarrow 0} \frac{2}{\pi^{2}} \frac{\epsilon}{\left(k^{2}+\epsilon\right)^{3}}=\delta(k) .
$$

Using the coherent state basis $\left|\xi_{1}, \xi_{2}\right\rangle=\left|\xi_{1}\right\rangle \otimes\left|\xi_{2}\right\rangle$ as in (4.28), it is easy to see that the total divergence in (4.47) vanishes on $\mathbf{S}^{3}$ in the large $k$ limit. So we have

$$
H_{\nu \nu}(x, y)=-\frac{1}{4 \pi^{2}}
$$

Finally we get

$$
\begin{aligned}
& -D_{\mu} D_{\mu}\left(v(x)^{\dagger} G^{(0)}(x, y) v(y)\right) \\
& =\delta(x-y)-\frac{1}{2 \pi^{2}}\left(v_{1}(x)^{\dagger} b_{1} \bar{\sigma}_{\mu} f_{1}(x) b_{1}^{\dagger} \otimes v_{2}(x)^{\dagger} b_{2} \bar{\sigma}_{\mu} f_{2}(x) b_{2}^{\dagger}\right) v(y) .
\end{aligned}
$$

Note that the above expression is exactly the same form as the commutative case.

Therefore, in order to get the answer (3.17) for the spin-1 propagator (4.34), we must show that

$$
\begin{aligned}
& -D_{\mu} D_{\mu} C(x, y)= \\
& 4\left(\left(v_{1}(x)^{\dagger} b_{1} f_{1}(x)\right)_{\alpha} \otimes\left(v_{2}(x)^{\dagger} b_{2} f_{2}(x)\right)_{\beta}\right)\left(\left(b_{1}^{\dagger} v_{1}(y)\right)_{\gamma} \otimes\left(b_{2}^{\dagger} v(y)\right)_{\delta}\right) \varepsilon_{\alpha \beta} \varepsilon_{\gamma \delta},
\end{aligned}
$$

where we used (3.3) and (4.33). Since the right-hand side of (4.51) has exactly the same form as the commutative one, we will take the same ansatz for $C(x, y)$ with commutative case [45]:

$$
C_{u s, v t}(x, y)=M_{i j, l m}\left(v_{1}(x)^{\dagger} b_{1}\right)_{u, i \alpha}\left(v_{2}(x)^{\dagger} b_{2}\right)_{s, j \beta}\left(b_{1}^{\dagger} v_{1}(y)\right)_{l \gamma, v}\left(b_{2}^{\dagger} v_{2}(y)\right)_{m \delta, t} \varepsilon_{\alpha \beta} \varepsilon_{\gamma \delta}
$$


or in the tensor notation

$$
C(x, y)=\left(\left(v_{1}(x)^{\dagger} b_{1}\right)_{\alpha} \otimes\left(v_{2}(x)^{\dagger} b_{2}\right)_{\beta}\right) M\left(\left(b_{1}^{\dagger} v_{1}(y)\right)_{\gamma} \otimes\left(b_{2}^{\dagger} v_{2}(y)\right)_{\delta}\right) \varepsilon_{\alpha \beta} \varepsilon_{\gamma \delta},
$$

where $M_{i j, l m}$ is a constant matrix to be determined later and $u, v=1, \cdots, N_{1}=\operatorname{dim} G_{1}, s, t=$ $1, \cdots, N_{2}=\operatorname{dim} G_{2}$ are group indices and $i, l=1, \cdots, k_{1}, j, m=1, \cdots, k_{2}$ are instanton number indices. Using the formula (see Eqs. (4.13) and (4.19) )

$$
\begin{aligned}
& D_{\mu} v_{a}(x)^{\dagger}=v_{a}(x)^{\dagger} b_{a} \bar{\sigma}_{\mu} f_{a}(x) \Delta_{a}(x)^{\dagger}, \\
& D_{\mu} D_{\mu} v_{a}(x)^{\dagger}=-4 v_{a}(x)^{\dagger} b_{a} f_{a}(x) b_{a}^{\dagger}
\end{aligned}
$$

and the multiplication (4.33), it is straightforward to show that

$$
\begin{aligned}
& -D_{\mu} D_{\mu} C(x, y)=4\left(\left(v_{1}(x)^{\dagger} b_{1} f_{1}(x)\right)_{\alpha} \otimes\left(v_{2}(x)^{\dagger} b_{2} f_{2}(x)\right)_{\beta}\right) \\
& \left(b_{1}^{\dagger} b_{1} \otimes f_{2}^{-1}(x)+f_{1}^{-1}(x) \otimes b_{2}^{\dagger} b_{2}-\left(\Delta_{1}(x)^{\dagger} b_{1}\right)_{\eta \xi} \otimes\left(\Delta_{2}(x)^{\dagger} b_{2}\right)_{\chi \zeta} \varepsilon_{\eta \chi} \varepsilon_{\xi \zeta}\right) M \\
& \left(\left(b_{1}^{\dagger} v_{1}(y)\right)_{\gamma} \otimes\left(b_{2}^{\dagger} v_{2}(y)\right)_{\delta}\right) \varepsilon_{\alpha \beta} \varepsilon_{\gamma \delta},
\end{aligned}
$$

where we used (3.3) at the final stage. Thus if the matrix $M$ satisfies

$$
\left(b_{1}^{\dagger} b_{1} \otimes f_{2}^{-1}(x)+f_{1}^{-1}(x) \otimes b_{2}^{\dagger} b_{2}-\left(\Delta_{1}(x)^{\dagger} b_{1}\right)_{\eta \xi} \otimes\left(\Delta_{2}(x)^{\dagger} b_{2}\right)_{\chi \zeta} \varepsilon_{\eta \chi} \varepsilon_{\xi \zeta}\right)=M^{-1}
$$

we finally prove Eq.(4.51) and so (3.17) for the tensor product $G_{1} \times G_{2}$ where the adjoint representation is a special case. However, to achieve this final goal, we should go further since Eq.(4.56) appears to state that the constant matrix $M$ is the inverse of an $x$-dependent matrix. In commutative space, as a result of conformal invariance of the matrix $M$ [45], the $x$-dependent parts of the left-hand side in (4.56) are completely canceled. We will show that it is also the case even for noncommutative space, but the matrix $M$ is slightly modified by the noncommutativity.

First note that, in the canonical basis (4.9),

$$
f_{a}^{-1}(x)=\Delta_{a}(x)^{\dagger} \Delta_{a}(x)=a_{a}^{\dagger} a_{a}-\xi_{a}^{\mu} x_{\mu}+x^{2}-\frac{1}{2} \sigma_{\mu \nu} \theta^{\mu \nu}
$$

and

$$
\begin{aligned}
\left(\Delta_{1}(x)^{\dagger} b_{1}\right)_{\eta \xi} \otimes\left(\Delta_{2}(x)^{\dagger} b_{2}\right)_{\chi \zeta} \varepsilon_{\eta \chi} \varepsilon_{\xi \zeta}= & \left(a_{1}^{\dagger} b_{1}\right)_{\eta \xi} \otimes\left(a_{2}^{\dagger} b_{2}\right)_{\chi \zeta} \varepsilon_{\eta \chi} \varepsilon_{\xi \zeta} \\
& -2 x_{\mu}\left(\xi_{1}^{\mu} \otimes 1\right)-2 x_{\mu}\left(1 \otimes \xi_{2}^{\mu}\right)+2 x^{2}
\end{aligned}
$$


Using these results, it is easy to see that the $x$-dependent parts of the left-hand side in (4.56) are completely canceled and the matrix $M$ is defined by

$$
\left(b_{1}^{\dagger} b_{1} \otimes\left(a_{2}^{\dagger} a_{2}-\frac{1}{2} \sigma_{\mu \nu} \theta^{\mu \nu}\right)+\left(a_{1}^{\dagger} a_{1}-\frac{1}{2} \sigma_{\mu \nu} \theta^{\mu \nu}\right) \otimes b_{2}^{\dagger} b_{2}-\left(a_{1}^{\dagger} b_{1}\right)_{\eta \xi} \otimes\left(a_{2}^{\dagger} b_{2}\right)_{\chi \zeta} \varepsilon_{\eta \chi} \varepsilon_{\xi \zeta}\right)=M^{-1} .
$$

Note that $a_{a}^{\dagger} a_{a}-\frac{1}{2} \sigma_{\mu \nu} \theta^{\mu \nu}$ is proportional to the identity matrix in quaternionic space while $a_{a}^{\dagger} a_{a}$ is not, as seen from (4.57). We see that the matrix $M$ is deformed by the noncommutativity, but only for non-BPS instanton background, that is anti-self-dual (self-dual) instantons in selfdual (anti-self-dual) $\mathbf{R}_{N C}^{4}$ and all instantons for (2.8), since $\eta_{\mu \nu}^{a} \bar{\eta}_{\mu \nu}^{b}=0$. If $\theta^{\mu \nu}=0$, of course, we recover the result in the commutative space.

In order to construct the propagator for $q \otimes \bar{q}$ in $(4.29)$, that is, the adjoint representation of $U(N)$, we take 45

$$
\begin{aligned}
& a_{1}=a, \quad a_{2}=a^{*} \sigma^{2}, \quad b_{1}=b, \quad b_{2}=b^{*} \sigma^{2}, \\
& v_{1}(x)=v_{2}(x)^{*}=v(x)
\end{aligned}
$$

and anti-hermitian generators of $U(N)$ as $T^{A}, A=1, \cdots, N^{2}$ which are normalized as

$$
\operatorname{tr}\left(T^{A} T^{B}\right)=-\frac{1}{2} \delta_{A B}
$$

where $T^{N^{2}}=\frac{1}{i \sqrt{2 N}} \mathbf{1}_{N}$. Then the propagator $G^{A B}(x, y)$ for the adjoint representation can be obtained by multiplying Eq.(4.34) by $T_{u s}^{A}, T_{v t}^{B}$ and summing over $u, s, v, t=1, \cdots, N$ :

$$
\begin{aligned}
G_{A B}(x, y)= & {\left[v(x)^{\dagger}\right]_{u, \lambda} T_{u s}^{A}[v(x)]_{\rho, s} G^{(0)}(x, y)[v(y)]_{\lambda, v} T_{v t}^{B}\left[v(y)^{\dagger}\right]_{t, \rho} } \\
& +\frac{1}{4 \pi^{2}} M_{i j, l m}\left[w(x)^{\dagger}\right]_{u, i \alpha} T_{u s}^{A}[w(x)]_{j \alpha, s}[w(y)]_{l \beta, v} T_{v t}^{B}\left[w(y)^{\dagger}\right]_{t, m \beta},
\end{aligned}
$$

where $\lambda, \rho=1, \cdots, N+2 k$ are ADHM indices and we introduced a $2 k \times N$ matrix $w(x)=b^{\dagger} v(x)$.

\section{Discussions}

We have explicitly constructed Green functions for a scalar field in an arbitrary representation of gauge group propagating in noncommutative instanton background. We have shown that the propagators in the adjoint representation are deformed by noncommutativity while those in the fundamental representation have exactly the same form as the commutative case.

We showed, generalizing the argument in 44 to noncommutative space, that the propagators for spinor and vector fields can be constructed in terms of those for the scalar field in noncommutative instanton background. However it was pointed out in [4] that the vector propagator suffers from an infrared divergence. Let's discuss this problem in our context. The 
vector propagator can be constructed by the operator expression (3.41) which is involved to the convolution integral over $z$-coordinates

$$
\left\langle x\left|\left(\frac{1}{D^{2}}\right)_{A B}^{2}\right| y\right\rangle=\operatorname{Tr}_{\mathcal{H}}^{z} G_{A C}(x, z) G_{C B}(z, y),
$$

where $G_{A B}(x, y)$ is defined by (4.62). Using the asymptotic behavior [53, 14] of several ADHM quantities at large $z$ limit, in which the noncommutativity of space is irrelevant, one can check that the integral (5.1) is logarithmically divergent or logarithmically divergent sum, e.g. $\sum_{n} n^{-1}$, in noncommutative case. According to [44], one can see that this divergence is coming from the zero mode fluctuations corresponding to global gauge rotations and an overall scale change, which have already been contained into the zero mode sum (3.29), where the zero mode for the scale change is generated by the Lorentz rotation of the gauge zero modes. However note that the global gauge zero modes are not normalizable on $\mathbf{R}^{4}$ or $\mathbf{R}_{N C}^{4}$ since it is noncompact space [54, 51]. A natural way to remove this logarithmic divergence is to put the theory on compactified Euclidean space, i.e. $\mathbf{S}^{4}$, as in [55, 56]. As shown in [56, this compactified Euclidean formalism provides a gauge invariant normalization for the global gauge zero modes since the volume of $\mathbf{S}^{4}$ is now finite. Thus the divergence in the vector propagator may be cured in this way because the convolution integral (5.1) on $\mathbf{S}^{4}$ can be finite. It will be interesting to see if the infrared divergence in the vector propagator can be cured by an "appropriate" compactification of noncommutative space in the same way.

Let's consider the massless Dirac equation defined by the covariant derivative (4.29) in the background of anti-self-dual instantons. In this case it has only positive chirality solutions 442] described by two spinors $\psi_{R}=\psi_{u s, \alpha}$ satisfying

$$
\sigma^{\mu} D_{\mu} \psi_{R}=0
$$

Take the same ansatz as the commutative case [45]

$$
\psi_{u s, \alpha}=\left[v_{1}(x)^{\dagger} b_{1} \sigma^{2} f_{1}(x)\right]_{u, i \alpha}\left[v_{2}(x)^{\dagger} d\right]_{s, i}+\left[v_{1}(x)^{\dagger} c\right]_{u, i}\left[v_{2}(x)^{\dagger} b_{2} \sigma^{2} f_{2}(x)\right]_{s, i \alpha},
$$

where $c, d$ are constant $\left(N_{1}+2 k_{1}\right) \times k_{2}$ and $\left(N_{2}+2 k_{2}\right) \times k_{1}$ matrices to be determined. Here we are using ordinary multiplication rather than the tensor product (4.33) since $c, d$ are coupling two spaces $G_{1}$ and $G_{2}$ together. In the case of adjoint representation, using (4.60), the ansatz (5.3) can be arranged into the form

$$
\psi_{R}=v(x)^{\dagger} \mathcal{M} f(x) b^{\dagger} v(x)-v(x)^{\dagger} b f(x) \mathcal{M}^{\dagger} v(x),
$$

with $(N+2 k) \times k$ matrix $\mathcal{M}$. Using the formula (4.54) and

$$
\bar{\sigma}^{\mu}\left(\Delta_{a}(x)^{\dagger} b_{\alpha}\right) \bar{\sigma}^{\mu}=-2 b_{a}^{\dagger} \Delta_{a}(x), \quad \sigma^{\mu}\left(b_{a}^{\dagger} \Delta_{a}(x)\right) \bar{\sigma}^{\mu}=2\left(\Delta_{a}(x)^{\dagger} b_{a}+b_{a}^{\dagger} \Delta_{a}(x)\right),
$$


it is straightforward to calculate Eq. (5.2)

$$
\begin{aligned}
\sigma^{\mu} D_{\mu} \psi_{R}= & 2 \varepsilon_{\beta \gamma}\left[v_{1}(x)^{\dagger} b_{1} f_{1}(x)\right]_{u, i \beta} \\
& \left(\left[v_{2}(x)^{\dagger} b_{2} f_{2}(x)\right]_{s, j \gamma}\left[\Delta_{2}(x)^{\dagger} d\right]_{j \alpha, i}-\left[\Delta_{1}(x)^{\dagger} c\right]_{i \alpha, j}\left[v_{2}(x)^{\dagger} b_{2} f_{2}(x)\right]_{s, j \gamma}\right) .
\end{aligned}
$$

In the commutative case [45], the equation (5.2) requires that

$$
\left[a_{1}^{\dagger} c\right]_{i \alpha, j}=\left[a_{2}^{\dagger} d\right]_{j \alpha, i}, \quad\left[b_{1}^{\dagger} c\right]_{i \alpha, j}=\left[b_{2}^{\dagger} d\right]_{j \alpha, i} .
$$

However, in the noncommutative space, we cannot say that the solution of (5.2) would be (5.7) since $\mathbf{x}$ does not necessarily commute with $v_{2}(x)^{\dagger} b_{2} f_{2}(x)$. So the simple minded ansatz (5.3) doesn't work for the noncommutative space. To find the fermionic zero modes for the gauge group $G_{1} \times G_{2}$, it may be needed to apply a systematic way for the tensor product of instantons as done in [45]. Unfortunately, the ADHM construction for the tensor product involves in tedious and complicated manipulations even for commutative space. We didn't succeed the generalization to the noncommutative space yet. Anyway we put this problem for future work.

In Sec. 4, we observed that the $x$-dependent matrix $M$ (4.56) is equal to the constant matrix $M$ (4.59) and the matrix $M$ is deformed by the noncommutativity only for non-BPS instantons. In commutative space, the $x$-independence of the matrix $M$ is a result of conformal invariance and the conformal invariance has an important role to calculate multi-instanton determinant [57, 58, 59, 60]. Since, for BPS instantons (commutative instantons are always BPS), the matrix $M$ has the same form as the commutative one, the conformal invariance for this background should be manifest. Although the matrix $M$ is deformed by the noncommutativity for non-BPS instantons, it is still a constant matrix. Thus one may expect (deformed) conformal invariance even for the non-BPS background. In [42, we observed that the conformal zero modes have a similar deformation by the noncommutativity and we speculated that the conformal symmetry has to act nontrivially only on the $S U(N)$ instanton sector. These deformations of conformal symmetry in zero modes and propagators should be related to each other.

Let's briefly discuss the conformal property of the matrix $M$ (4.59) in the BPS background, in which the $\sigma_{\mu \nu} \theta^{\mu \nu}$ term vanishes. From Eq.(4.56) and Eq.(4.59), we see that the matrix $M$ in (4.59) is invariant under the transformations

$$
a_{a} \rightarrow a_{a}+b_{a} \overline{\mathbf{p}}, \quad b_{a} \rightarrow b_{a}, \quad a=1,2 .
$$

Since it is symmetric under interchange of $a_{a}$ and $b_{a}$, it is also invariant under the transformations (use $\sigma^{\mu} \sigma^{\nu}+\bar{\sigma}^{\mu} \bar{\sigma}^{\nu}=\operatorname{tr}_{2}\left(\sigma^{\mu} \sigma^{\nu}\right)=\operatorname{tr}_{2}\left(\bar{\sigma}^{\mu} \bar{\sigma}^{\nu}\right)$ to check)

$$
a_{a} \rightarrow a_{a}, \quad b_{a} \rightarrow b_{a}+a_{a} \overline{\mathbf{p}} .
$$

While, under the transformations

$$
a_{a} \rightarrow a_{a} \overline{\mathbf{p}}, \quad b_{a} \rightarrow b_{a} \overline{\mathbf{q}}
$$


it changes by a factor $p^{2} q^{2}$ for any quaternions $\overline{\mathbf{p}}, \overline{\mathbf{q}}$ (use (3.2) to check). This factor can be scaled to unity in terms of simultaneous global scaling of $a_{a}, b_{a}$ by a real number. The above transformations (5.8)-(5.10) actually correspond to unimodular conformal group [45] (so fifteen parameter group).

If we try to generalize the above consideration to the non-BPS instantons, in which we have the $\sigma_{\mu \nu} \theta^{\mu \nu}$ term, we immediately meet some nontrivial problems. The main source of this difficulty is that the matrix $M(4.59)$ is asymmetric under interchange of $a_{a}$ and $b_{a}$ due to the presence of the inhomogeneous term, i.e. $\sigma_{\mu \nu} \theta^{\mu \nu}$. The scale transformation (5.10) doesn't generate an overall scale either because of the inhomogeneous term, thus some modified transformation would be genuinely required. At current stage, we don't know how to modify the conformal transformations (5.8)-(5.10). We hope to report some progress along this line in near future.

\section{Acknowledgments}

We thank Keun-Young Kim for helpful discussions and careful reading of this manuscript. BHL is supported by the Ministry of Education, BK21 Project No. D-0055 and by grant No. R011999-00018 from the Interdisciplinary Research Program of the KOSEF. HSY is supported by NSC (NSC90-2811-M-002-019). He also acknowledges NCTS as well as CTP at NTU for partial support. 


\section{References}

[1] A. A. Belavin, A. M. Polyakov, A. S. Schwartz and Yu. S. Tyupkin, Phys. Lett. 59B, 85 (1975).

[2] R. Jackiew and C. Rebbi, Phys. Rev. Lett. 37, 172 (1976).

[3] C. G. Callan, R. F. Dashen and D. J. Gross, Phys. Lett. 63B, 334 (1976).

[4] Instantons in Gauge Theories, edited by M. Shifman, (World Scientific, Singapore, 1994).

[5] G. 't Hooft, Phys. Rev. Lett. 37, 8 (1976).

[6] G. 't Hooft, Phys. Rev. D14, 3432 (1976); ibid. (E) D18, 2199 (1978).

[7] For a recent review, for example, see T. Schafer and E. V. Shuryak, Rev. Mod. Phys. 70, 323 (1998), hep-ph/9610451.

[8] E. Witten, Nucl. Phys. B460, 541 (1996), hep-th/9511030.

[9] M. R. Douglas, Branes within Branes, hep-th/9512077; J. Geom. Phys. 28, 255 (1998), hep-th/9604198.

[10] A. Strominger and C. Vafa, Phys. Lett. B379, 99 (1996), hep-th/9601029.

[11] C. G. Callan and J. M. Maldacena, Nucl. Phys. B472, 591 (1996), hep-th/9602043.

[12] T. Banks and M. B. Green, J. High Energy Phys. 05, 002 (1998), hep-th/9804170.

[13] M. Bianchi, M. B. Green, S. Kovacs and G. Rossi, J. High Energy Phys. 08, 013 (1998), hep-th/9807033.

[14] N. Dorey, T. J. Hollowood, V. V. Khoze, M. P. Mattis and S. Vandoren, Nucl. Phys. B552, 88 (1999), hep-th/9901128.

[15] For a review on Yang-Mills and D-instantons, see A. V. Belitsky, S. Vandoren and P. van Nieuwenhuizen, Class. Quant. Grav. 17, 3521 (2000), hep-th/0004186.

[16] N. A. Nekrasov and A. Schwarz, Comm. Math. Phys. 198, 689 (1998), hep-th/9802068.

[17] N. Seiberg and E. Witten, J. High Energy Phys. 09, 032 (1999), hep-th/9908142.

[18] M. F. Atiyah, N. J. Hitchin, V. G. Drinfeld and Yu. I. Manin, Phys. Lett. 65A, 185 (1978).

[19] K. Lee and P. Yi, Phys. Rev. D61, 125015 (2000), hep-th/9911186. 
[20] H. W. Braden and N. A. Nekrasov, Spacetime Foam from Noncommutative Instantons, hep-th/9912019.

[21] K. Furuuchi, Prog. Theor. Phys. 103, 1041 (2000), hep-th/9912047.

[22] A. Kapustin, A. Kuznetsov and D. Orlov, Comm. Math. Phys. 221, 385 (2001), hep-th/0002193.

[23] P.-M. Ho, Twisted Bundle on Noncommuative Space and $U(1)$ Instanton, hep-th/0003012.

[24] K.-Y. Kim, B.-H. Lee, and H. S. Yang, Comments on Instantons on Noncommutative $\mathbf{R}^{4}$, hep-th/0003093.

[25] K. Furuuchi, Comm. Math. Phys. 217, 579 (2001), hep-th/0005199.

[26] K. Lee, D. Tong and S. Yi, Phys. Rev. D63, 065017 (2001), hep-th/0008092.

[27] K. Furuuchi, J. High Energy Phys. 03, 223 (2001), hep-th/0010119.

[28] A. Schwarz, Comm. Math. Phys. 221, 433 (2001), hep-th/0102182.

[29] D. H. Correa, G. S. Lozano, E. F. Moreno and F. A. Schaposnik, Phys. Lett. B515, 206 (2001), hep-th/0105085.

[30] C.-S. Chu, V. V. Khoze and G. Travaglini, Nucl. Phys. B621, 101 (2002), hep-th/0108007.

[31] M. Hamanaka, Phys. Rev. D65, 085022 (2002), hep-th/0109070.

[32] T. Ishikawa, S.-I. Kuroki and A. Sako, J. High Energy Phys. 11, 068 (2001), hep-th/0109111.

[33] K.-Y. Kim, B.-H. Lee, and H. S. Yang, Phys. Lett. B523, 357 (2001), hep-th/0109121.

[34] O. Lechtenfeld and A. D. Popov, J. High Energy Phys. 03, 040 (2002), hep-th/0109209.

[35] T. Ishikawa, S.-I. Kuroki and A. Sako, Instanton Number Calculus on Noncommutative $\mathbf{R}^{4}$, hep-th/0201196.

[36] N. Dorey, T. J. Hollowood and V. V. Khoze, J. High Energy Phys. 03, 040 (2001), hep-th/0011247.

[37] T. J. Hollowood, V. V. Khoze and G. Travaglini, J. High Energy Phys. 05, 051 (2001), hep-th/0102045. 
[38] N. Dorey, T. J. Hollowood and V. V. Khoze, Notes on Soliton Bound-State Problems in Gauge Theory and String Theory, hep-th/0105090.

[39] C. Kim, K. Lee and P. Yi, Phys. Rev. D65, 065024 (2002), hep-th/0109217.

[40] T. J. Hollowood, J. High Energy Phys. 03, 038 (2002), hep-th/0201075.

[41] N. A. Nekrasov, Lectures on Open Strings and Noncommutative Gauge Theories, hep-th/0203109.

[42] K.-Y. Kim, B.-H. Lee and H. S. Yang, Zero-Modes and Atiyah-Singer Index in Noncommutative Instantons, to appear in Phys. Rev. D, hep-th/0205010.

[43] Y. Hiraoka, BPS Solutions of Noncommutative Gauge Theories in Four and Eight Dimensions, hep-th/0205283.

[44] L. S. Brown, R. D. Carlitz, D. B. Creamer and C. Lee, Phys. Rev. D17, 1583 (1978).

[45] E. F. Corrigan, P. Goddard and S. Templeton, Nucl. Phys. B151, 93 (1979).

[46] S. Cho, R. Hinterding, J. Madore and H. Steinacker, Int. J. Mod. Phys. D9, 161 (2000), hep-th/9903239.

[47] D. J. Gross and N. A. Nekrasov, J. High Energy Phys. 07, 034 (2000), hep-th/0005204.

[48] R. Gopakumar, S. Minwalla and A. Strominger, J. High Energy Phys. 05, 020 (2000), hep-th/0003160.

[49] For example, see Chapter 3 in N. N. Bogoliubov and D. V. Shirkov, Introduction to The Theory of Quantized Fields, 3rd ed., (John Wiley \& Sons, New York, 1980).

[50] E. F. Corrigan, D. B. Fairlie, P. Goddard and S. Templeton, Nucl. Phys. B140, 31 (1978).

[51] N. H. Christ, N. K. Stanton and E. J. Weinberg, Phys. Rev. D18, 2013 (1978).

[52] N. E. Wegge-Olsen, K-Theory and $C^{*}$-Algebras, (Oxford University Press, Oxford, 1993).

[53] E. Corrigan and P. Goddard, Ann. Phys. 154, 253 (1984).

[54] C. W. Bernard, N. H. Christ, A. H. Guth and E. J. Weinberg, Phys. Rev. D16, 2967 (1977).

[55] R. Jackiew and C. Rebbi, Phys. Rev. D14, 517 (1976).

[56] N. K. Nielsen and B. Schroer, Nucl. Phys. B127, 493 (1977).

[57] E. Corrigan, P. Goddard, H. Osborn and S. Templeton, Nucl. Phys. B159, 469 (1979). 
[58] H. Osborn, Nucl. Phys. B159, 1979 (497).

[59] B. Berg and M. Lüscher, Nucl. Phys. B160, 281 (1979).

[60] I. Jack, Nucl. Phys. B174, 526 (1980). 